\title{
ON THE GUTMAN INDEX OF THORN GRAPHS
}

\author{
Mahdieh Azari* \\ Department of Mathematics, Kazerun Branch, Islamic Azad University, \\ P. O. Box: 73135-168, Kazerun, Iran \\ *Corresponding author; E-mails: azari@kau.ac.ir and mahdie.azari@gmail.com
}

(Received July 24, 2017; Accepted August 08, 2017)

\begin{abstract}
In this paper, the relation between the Gutman index of a simple connected graph and its thorn graph is stablished and several special cases of the result are examined. Results are applied to compute the Gutman index of thorn paths, thorn rods, caterpillars, thorn rings, thorn stars, Kragujevac trees, and dendrimers.
\end{abstract}

Keywords: Gutman index, degree distance, terminal Wiener index, thorn graph, dendrimer.

\section{INTRODUCTION}

Let $G$ be an $n$-vertex simple connected graph with vertex set $V(G)=\left\{v_{1}, v_{2}, \ldots, v_{n}\right\}$ and let $\mathrm{P}=\left(p_{1}, p_{2}, \ldots, p_{n}\right)$ be an $n$-tuple of nonnegative integers. The thorn graph $G_{\mathrm{P}}$ is the graph obtained by attaching $p_{i}$ pendent vertices (terminal vertices or vertices of degree one) to the vertex $v_{i}$ of $G$, for $i=1,2, \ldots, n$ (see Fig. 1 ).

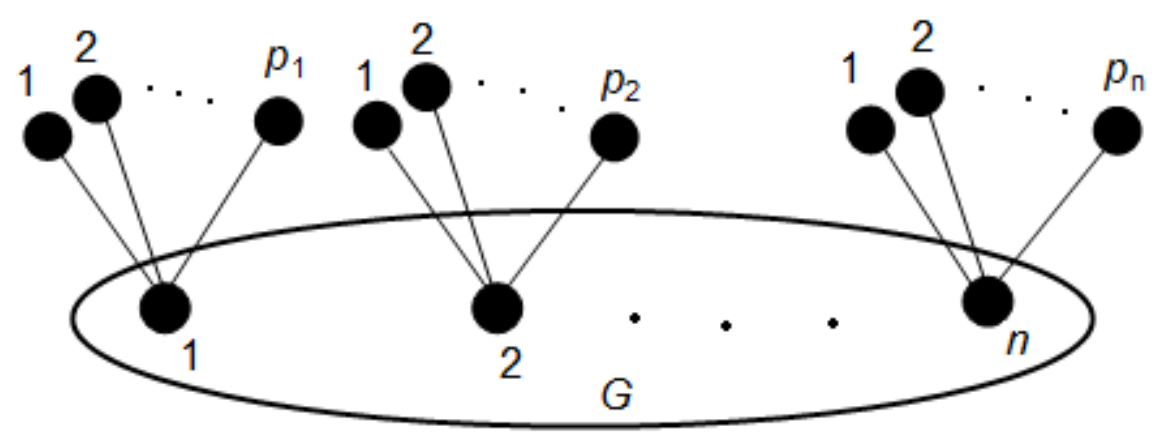

Figure 1. The thorn graph $G_{\mathrm{P}}$ with parameters $p_{1}, p_{2}, \ldots, p_{n}$.

The $p_{i}$ pendent vertices attached to the vertex $v_{i}$ are called thorns of $v_{i}$. We denote the set of $p_{i}$ thorns of $v_{i}$ by $V_{i}, i=1,2, \ldots, n$. Clearly, $V\left(G_{\mathrm{P}}\right)=V(G) \cup V_{1} \cup V_{2} \cup \ldots \cup V_{n}$. The concept of thorn graphs was introduced by GUTMAN (1998) and eventually found a variety of chemical applications; see (BYTAUTAS et al., 2001; BONCHEV and KLEIN, 2002; VUKIČEviĆ and 
GRAOVAC, 2004; ZHOU, 2005; VUKIČEVIĆ et al., 2005, 2007; W ALIKAR et al., 2006; KLEIN et al., 2007; HeYdARI and GUTMAN, 2010; Li, 2011; AlizAdeH et al., 2014; AZARI, 2014; AZARI and IRANMANESH, 2015, 2016). The motivation for the study of thorn graphs came from a particular case, namely $G_{\mathrm{P}}=G_{\left(\gamma-\gamma_{1}, \gamma-\gamma_{2}, \ldots, \gamma-\gamma_{n}\right)}$, where $\gamma_{i}$ is the degree of the $i$-th vertex of $G$ and $\gamma$ is a constant $\left(\gamma \geq \gamma_{i}\right.$ for all $\left.i=1,2, \ldots, n\right)$. Then the vertices of $G_{\mathrm{P}}$ are either of degree $\gamma$ or of degree one. If in addition $\gamma=4$, then the thorn graph $G_{\mathrm{P}}$ is just what CAYLEY (1874) calls a plerogram (a graph in which every atom is represented by a vertex and adjacent atoms are connected by a chemical bond) and POLYA (1937) a $C-H$ graph. The parent graph $G$ would then be referred to as a kenogram (CAYLEY, 1874) (a graph obtained from a plerogram by suppressing hydrogen atoms) or a $C$-graph (POLYA, 1937). The plerogram and kenogram of 2,3,3-trimethylpentane are depicted in Fig. 2.

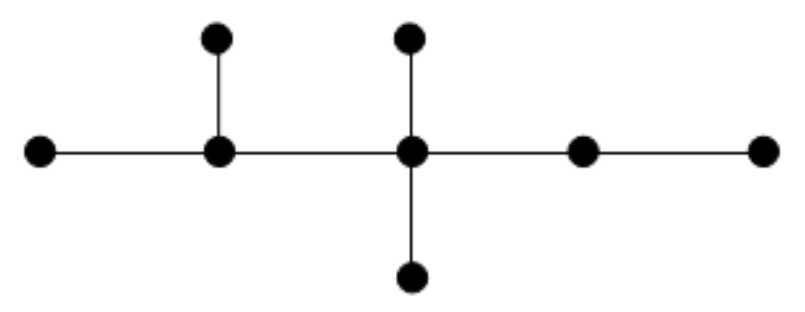

(a)

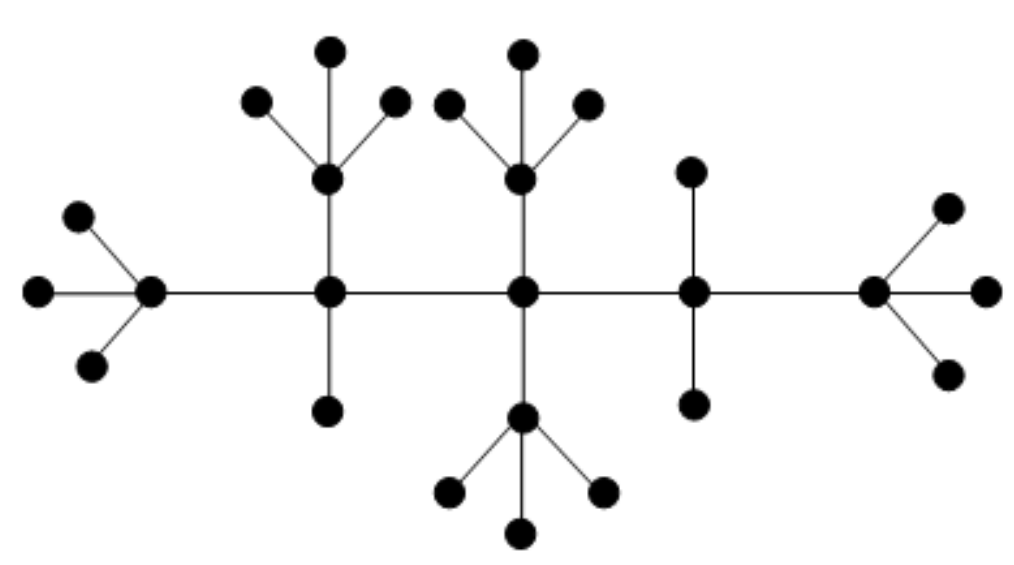

(b)

Figure 2. (a) The kenogram of 2,3,3-trimethylpentane, (b) The plerogram of 2,3,3-trimethylpentane.

A topological index is a numeric quantity that is mathematically derived in a direct and unambiguous manner from the structural graph of a molecule. It is used in theoretical chemistry for the design of chemical compounds with given physico-chemical properties or given pharmacologic and biological activities (DIUDEA, 2001). It is well known that the study of topological indices of kenograms is much more conventional than plerograms, because of their simplicity and the fact that many topological indices give highly correlated results on plerograms and kenograms (GUTMAN et al., 1998). The study of thorn graphs unifies these two approaches by giving mathematical formulae that connect the values of topological indices of kenograms and plerograms.

In this paper, we study relation between the Gutman index of a simple connected graph and its thorn graph and apply the results to compute the Gutman index of thorn paths, thorn rods, caterpillars, thorn rings, thorn stars, Kragujevac trees, and dendrimers. 


\section{DEFINITIONS AND PRELIMINARIES}

In this paper, we consider connected finite graphs without any loops or multiple edges. The best known and widely used topological index is the Wiener index introduced by WIENER (1947), who used it for modeling the shape of organic molecules and for calculating several of their physico-chemical properties. The Wiener index of a graph $G$ is defined as the sum of distances between all pairs of vertices of $G$,

$$
W(G)=\sum_{\{u, v\} \subseteq V(G)} d_{G}(u, v),
$$

where $d_{G}(u, v)$ denotes the distance between the vertices $u$ and $v$ in $G$.

The degree distance was introduced by DOBRYNIN and KocheTOVA (1994) and at the same time by GUTMAN (1994) as a weighted version of the Wiener index. The degree distance of a graph $G$ is defined as

$$
D D(G)=\sum_{\{u, v\} \subseteq V(G)}\left[d_{G}(u)+d_{G}(v)\right] d_{G}(u, v) .
$$

In fact, if $T$ is a tree on $n$ vertices, the Wiener index and degree distance are closely related by $D D(T)=4 W(T)-n(n-1)$; see (GUTMAN, 1994).

The Gutman index (also known as Schultz index of the second kind) was introduced by GuTMAN (1994) as a kind of vertex-valency-weighted sum of the distances between all pairs of vertices in a graph. Gutman revealed that in the case of acyclic structures, the index is closely related to the Wiener index and reflects precisely the same structural features of a molecular as the Wiener index does. The Gutman index of a graph $G$ is defined as

$$
\operatorname{Gut}(G)=\sum_{\{u, v\} \subseteq V(G)} d_{G}(u) d_{G}(v) d_{G}(u, v) .
$$

We refer the reader to (FENG and LiU, 2011; ANDOVA et al., 2012; CHEN, 2016; KNOR et al., 2014; GutMan, 2016; AZARI, 2016; AZARI and DiVANPOUR, 2017) for more information on the Gutman index and degree distance.

The concept of terminal Wiener index was put forward by GUTMAN et al. (2009). Somewhat later, but independently, SzÉKELY et al. (2011) arrived at the same idea. The terminal Wiener index $T W(G)$ of a graph $G$ is defined as the sum of distances between all pairs of its pendent vertices,

$$
T W(G)=\sum_{\{u, v\} \subseteq V^{\prime}(G)} d_{G}(u, v) .
$$

where $V^{\prime}(G)$ is the set of all pendent vertices of $G$.

For $u \in V(G)$, we define the quantity $T W_{G}(u)$ as the sum of distances between $u$ and all pendent vertices of $G$,

$$
T W_{G}(u)=\sum_{v \in V^{\prime}(G)} d_{G}(u, v)
$$

It is easy to see that, $T W(G)=\frac{1}{2} \sum_{u \in V^{\prime}(G)} T W_{G}(u)$. 


\section{RESULTS AND DISCUSSION}

In this section, we establish relation between the Gutman index of a simple connected graph $G$ and its thorn graph $G_{\mathrm{P}}$, and examine several special cases of the result.

Theorem 1. Let $G$ be a connected $n$-vertex graph with vertex set $V(G)=\left\{v_{1}, v_{2}, \ldots, v_{n}\right\}$, and let $G_{\mathrm{P}}$ be the thorn graph of $G$ with nonnegative parameters $p_{1}, p_{2}, \ldots, p_{n}$. Then

$$
\begin{aligned}
\operatorname{Gut}\left(G_{\mathrm{P}}\right)= & \operatorname{Gut}(G)+2 \sum_{1 \leq i<j \leq n}\left(p_{j} d_{G}\left(v_{i}\right)+p_{i} d_{G}\left(v_{j}\right)\right) d_{G}\left(v_{i}, v_{j}\right)+4 \sum_{1 \leq i<j \leq n} p_{i} p_{j} d_{G}\left(v_{i}, v_{j}\right) \\
& +2\left(\sum_{i=1}^{n} p_{i}\right)^{2}+(2|E(G)|-1) \sum_{i=1}^{n} p_{i} .
\end{aligned}
$$

Proof. By definition of the Gutman index, we have

$$
\operatorname{Gut}\left(G_{\mathrm{P}}\right)=\sum_{\{u, v\} \subseteq V\left(G_{\mathrm{P}}\right)} d_{G_{\mathrm{P}}}(u) d_{G_{\mathrm{P}}}(v) d_{G_{\mathrm{P}}}(u, v) .
$$

By definition of the graph $G_{\mathrm{P}}$, the above sum can be partitioned into four sums as follows.

The first sum $S_{1}$ consists of contriutions to $\operatorname{Gut}\left(G_{\mathrm{P}}\right)$ of pairs of vertices from $G$,

$$
\begin{aligned}
S_{1} & =\sum_{1 \leq i<j \leq n} d_{G_{\mathrm{P}}}\left(v_{i}\right) d_{G_{\mathrm{P}}}\left(v_{j}\right) d_{G_{\mathrm{P}}}\left(v_{i}, v_{j}\right) \\
& =\sum_{1 \leq i<j \leq n}\left(d_{G}\left(v_{i}\right)+p_{i}\right)\left(d_{G}\left(v_{j}\right)+p_{j}\right) d_{G}\left(v_{i}, v_{j}\right) \\
& =\operatorname{Gut}(G)+\sum_{1 \leq i<j \leq n}\left(p_{j} d_{G}\left(v_{i}\right)+p_{i} d_{G}\left(v_{j}\right)\right) d_{G}\left(v_{i}, v_{j}\right)+\sum_{1 \leq i<j \leq n} p_{i} p_{j} d_{G}\left(v_{i}, v_{j}\right) .
\end{aligned}
$$

The second sum $S_{2}$ consists of contriutions to $\operatorname{Gut}\left(G_{\mathrm{P}}\right)$ of pairs of vertices from $V_{i}$ for all $1 \leq i \leq n$,

$$
S_{2}=\sum_{i=1}^{n} \sum_{\{u, v\} \subseteq V_{i}} d_{G_{\mathrm{P}}}(u) d_{G_{\mathrm{P}}}(v) d_{G_{\mathrm{P}}}(u, v)=\sum_{i=1}^{n} \sum_{\{u, v\} \subseteq V_{i}} 1 \times 1 \times 2=2 \sum_{i=1}^{n}\left(\begin{array}{c}
p_{i} \\
2
\end{array}\right)=\sum_{i=1}^{n} p_{i}^{2}-\sum_{i=1}^{n} p_{i} .
$$

The third sum $S_{3}$ is taken over all pairs of vertices such that one of them, $u$, is in $G$, and the other one, $v$, is in $V_{j}$ for $1 \leq j \leq n$. So

$$
\begin{aligned}
S_{3} & =\sum_{i=1}^{n} \sum_{j=1}^{n} \sum_{u=v_{i}} \sum_{v \in V_{j}} d_{G_{\mathrm{P}}}(u) d_{G_{\mathrm{P}}}(v) d_{G_{\mathrm{P}}}(u, v) \\
& =\sum_{i=1}^{n} \sum_{j=1}^{n} \sum_{u=v_{i}} \sum_{i \in V_{j}}\left(d_{G}\left(v_{i}\right)+p_{i}\right) \times 1 \times\left(d_{G}\left(v_{i}, v_{j}\right)+1\right) \\
& =\sum_{i=1}^{n} d_{G}\left(v_{i}\right) \sum_{j=1}^{n} p_{j} d_{G}\left(v_{i}, v_{j}\right)+\sum_{i=1}^{n} d_{G}\left(v_{i}\right) \sum_{j=1}^{n} p_{j}+\sum_{i=1}^{n} p_{i} \sum_{j=1}^{n} p_{j} d_{G}\left(v_{i}, v_{j}\right)+\sum_{i=1}^{n} p_{i} \sum_{j=1}^{n} p_{j} .
\end{aligned}
$$

It is easy to check that

$$
\sum_{i=1}^{n} d_{G}\left(v_{i}\right) \sum_{j=1}^{n} p_{j} d_{G}\left(v_{i}, v_{j}\right)=\sum_{1 \leq i<j \leq n}\left(p_{j} d_{G}\left(v_{i}\right)+p_{i} d_{G}\left(v_{j}\right)\right) d_{G}\left(v_{i}, v_{j}\right) .
$$


Hence

$$
S_{3}=\sum_{1 \leq i<j \leq n}\left(p_{j} d_{G}\left(v_{i}\right)+p_{i} d_{G}\left(v_{j}\right)\right) d_{G}\left(v_{i}, v_{j}\right)+2 \sum_{1 \leq i<j \leq n} p_{i} p_{j} d_{G}\left(v_{i}, v_{j}\right)+\left(\sum_{i=1}^{n} p_{i}\right)^{2}+2|E(G)| \sum_{i=1}^{n} p_{i} .
$$

The fourth sum $S_{4}$ is taken over all pairs of vertices such that one of them, $u$, is in $V_{i}$, and the other one, $v$, is in $V_{j}$, where $1 \leq i \neq j \leq n$. So

$$
\begin{aligned}
S_{4} & =\sum_{1 \leq i<j \leq n} \sum_{u \in V_{i}} \sum_{v \in V_{j}} d_{G_{\mathrm{P}}}(u) d_{G_{\mathrm{P}}}(v) d_{G_{\mathrm{P}}}(u, v)=\sum_{1 \leq i<j \leq n} \sum_{u \in V_{i}} \sum_{v \in V_{j}} 1 \times 1 \times\left(d_{G}\left(v_{i}, v_{j}\right)+2\right) \\
& =\sum_{1 \leq i<j \leq n} p_{i} p_{j} d_{G}\left(v_{i}, v_{j}\right)+2 \sum_{1 \leq i<j \leq n} p_{i} p_{j} .
\end{aligned}
$$

It is easy to check that

$$
2 \sum_{1 \leq i<j \leq n} p_{i} p_{j}=\sum_{i=1}^{n} \sum_{j=1}^{n} p_{i} p_{j}-\sum_{i=1}^{n} p_{i}^{2}=\left(\sum_{i=1}^{n} p_{i}\right)^{2}-\sum_{i=1}^{n} p_{i}^{2}
$$

Hence

$$
S_{4}=\sum_{1 \leq i<j \leq n} p_{i} p_{j} d_{G}\left(v_{i}, v_{j}\right)+\left(\sum_{i=1}^{n} p_{i}\right)^{2}-\sum_{i=1}^{n} p_{i}^{2} .
$$

Eq. (1) is obtained by adding $S_{1}, S_{2}, S_{3}, S_{4}$, and simplifying the resulting expression.

For every connected graph $G$, we define

$$
\Psi(G)=\sum_{u \in V(G)-V^{\prime}(G)} d_{G}(u) T W_{G}(u) .
$$

In the following theorem, we find a formula for $\Psi\left(G_{P}\right)$.

Theorem 2. Let $G$ be a connected n-vertex graph with vertex set $V(G)=\left\{v_{1}, v_{2}, \ldots, v_{n}\right\}$, and let $G_{\mathrm{P}}$ be the thorn graph of $G$ with parameters $p_{1}, p_{2}, \ldots, p_{n}$ such that for every pendent vertex $v_{i}$ of $G, p_{i}>0$. Then

$$
\Psi\left(G_{P}\right)=\sum_{1 \leq i<j \leq n}\left(p_{j} d_{G}\left(v_{i}\right)+p_{i} d_{G}\left(v_{j}\right)\right) d_{G}\left(v_{i}, v_{j}\right)+2 \sum_{1 \leq i<j \leq n} p_{i} p_{j} d_{G}\left(v_{i}, v_{j}\right)+\left(\sum_{i=1}^{n} p_{i}\right)^{2}+2|E(G)| \sum_{i=1}^{n} p_{i} .
$$

Proof. Since for every pendent vertex $v_{i}$ of $G, p_{i}>0$, so $V^{\prime}\left(G_{P}\right)=V_{1} \cup V_{2} \cup \ldots \cup V_{n}$ and $V\left(G_{P}\right)-V^{\prime}\left(G_{P}\right)=V(G)$. Then

$$
\Psi\left(G_{P}\right)=\sum_{u \in V(G)} d_{G_{P}}(u) T W_{G_{P}}(u)=\sum_{u \in V(G)} \sum_{v \in V^{\prime}\left(G_{P}\right)} d_{G_{P}}(u) d_{G_{P}}(v) d_{G_{P}}(u, v) .
$$

One can easily see that, $\Psi\left(G_{P}\right)$ is the contribiutions to $\operatorname{Gut}\left(G_{\mathrm{P}}\right)$ of all pairs of vertices $\{u, v\}$ of $G_{\mathrm{P}}$ such that one of them, $u$, is in $G$, and the other one, $v$, is in $V_{j}$ for $1 \leq j \leq n$. So $\Psi\left(G_{P}\right)$ is equal to the sum $S_{3}$ in the proof of Theorem 1. Now using Eq. (2), we can get Eq. (3).

As a direct consequence of Theorem 2, we get the following corollary which will be used in the next section.

Corollary 1. Let $G$ be a connected $n$-vertex graph with $k$ pendent vertices, and let $G_{\mathrm{P}}$ be the thorn graph of $G$ obtained by attaching $p>0$ pendent vertices to each pendent vertex of $G$. Then

$$
\Psi\left(G_{P}\right)=2 p(p+1) T W(G)+p \Psi(G)+k p(k p+2|E(G)|) .
$$


Proof. Let $V(G)=\left\{v_{1}, v_{2}, \ldots, v_{n}\right\}$, and without loss of generality let $V^{\prime}(G)=\left\{v_{1}, v_{2}, \ldots, v_{k}\right\}$. By setting $p_{1}=p_{2}=\ldots=p_{k}=p$ and $p_{k+1}=p_{k+2}=\ldots=p_{n}=0$ in Eq. (3), we obtain

$$
\begin{aligned}
\Psi\left(G_{P}\right)= & \sum_{1 \leq i<j \leq k}(p \times 1+p \times 1) d_{G}\left(v_{i}, v_{j}\right)+\sum_{k+1 \leq i<j \leq n}\left[0 \times d_{G}\left(v_{i}\right)+0 \times d_{G}\left(v_{j}\right)\right] d_{G}\left(v_{i}, v_{j}\right) \\
& +\sum_{i=1}^{k} \sum_{j=k+1}^{n}\left[0 \times d_{G}\left(v_{i}\right)+p \times d_{G}\left(v_{j}\right)\right] d_{G}\left(v_{i}, v_{j}\right)+2 \sum_{1 \leq i<j \leq k} p^{2} d_{G}\left(v_{i}, v_{j}\right) \\
& +2 \sum_{k+1 \leq i<j \leq n} 0^{2} d_{G}\left(v_{i}, v_{j}\right)+2 \sum_{i=1}^{k} \sum_{j=k+1}^{n} p \times 0 \times d_{G}\left(v_{i}, v_{j}\right)+(k p)^{2}+2 k p \mid E(G) .
\end{aligned}
$$

Using the relations $\sum_{1 \leq i<j \leq k} d_{G}\left(v_{i}, v_{j}\right)=T W(G)$ and $\sum_{i=1}^{k} \sum_{j=k+1}^{n} d_{G}\left(v_{j}\right) d_{G}\left(v_{i}, v_{j}\right)=\Psi(G)$, we get Eq. (4).

Now, we express some special cases of Theorem 1.

Corollary 2. Let $G$ be a connected $n$-vertex graph, and let $G_{\mathrm{P}}$ be the thorn graph of $G$ with parameters $p_{1}=p_{2}=\ldots=p_{n}=p$, where $p$ is a nonnegative integer. Then

$$
G u t\left(G_{\mathrm{P}}\right)=G u t(G)+2 p D D(G)+4 p^{2} W(G)+n p(2 n p+2|E(G)|-1) .
$$

Corollary 3. Let $G$ be a connected $n$-vertex graph with $k$ pendent vertices, and let $G_{\mathrm{P}}$ be the thorn graph of $G$ obtained by attaching $p \geq 0$ pendent vertices to each pendent vertex of $G$. Then

$$
\operatorname{Gut}\left(G_{\mathrm{P}}\right)=\operatorname{Gut}(G)+4 p(p+1) T W(G)+2 p \Psi(G)+k p(2 k p+2|E(G)|-1) .
$$

Proof. Let $V(G)=\left\{v_{1}, v_{2}, \ldots, v_{n}\right\}$, and without loss of generality let $V^{\prime}(G)=\left\{v_{1}, v_{2}, \ldots, v_{k}\right\}$. By setting $p_{1}=p_{2}=\ldots=p_{k}=p$ and $p_{k+1}=p_{k+2}=\ldots=p_{n}=0$ in Eq. (1), we can get Eq. (6).

Corollary 4. Let $G$ be a connected $n$-vertex graph with vertex set $V(G)=\left\{v_{1}, v_{2}, \ldots, v_{n}\right\}$, and let $G_{\mathrm{P}}$ be the thorn graph of $G$ with parameters $p_{1}, p_{2}, \ldots, p_{n}$, where $p_{i}=d_{G}\left(v_{i}\right), i=1,2, \ldots, n$. Then

$$
\operatorname{Gut}\left(G_{\mathrm{P}}\right)=9 \operatorname{Gut}(G)+12|E(G)|^{2}-2|E(G)| .
$$

Corollary 5. Let $G$ be a connected $n$-vertex graph with vertex set $V(G)=\left\{v_{1}, v_{2}, \ldots, v_{n}\right\}$, and let $\gamma$ be an integer with the property $\gamma \geq d_{G}\left(v_{i}\right)$, for $i=1,2, \ldots, n$. Let $G_{\mathrm{P}}$ be the thorn graph of $G$ with parameters $p_{1}, p_{2}, \ldots, p_{n}$, where $p_{i}=\gamma-d_{G}\left(v_{i}\right), i=1,2, \ldots, n$. Then

$$
\operatorname{Gut}\left(G_{\mathrm{P}}\right)=\operatorname{Gut}(G)+4 \gamma^{2} W(G)-2 \gamma D D(G)+(n \gamma-2|E(G)|)(2 n \gamma-2|E(G)|-1) .
$$

Proof. It is easy to see that

$$
\begin{gathered}
\sum_{i=1}^{n} p_{i}=n \gamma-2|E(G)|, \sum_{1 \leq i<j \leq n}\left(p_{j} d_{G}\left(v_{i}\right)+p_{i} d_{G}\left(v_{j}\right)\right) d_{G}\left(v_{i}, v_{j}\right)=\gamma D D(G)-2 G u t(G), \\
\sum_{1 \leq i<j \leq n} p_{i} p_{j} d\left(v_{i}, v_{j} \mid G\right)=\gamma^{2} W(G)-\gamma D D(G)+\operatorname{Gut}(G) .
\end{gathered}
$$

Now using Eq. (1), we can get the desired result. 


\section{APPLICATIONS}

In this section, we apply the results of the previous section to compute the Gutman index of various classes of chemical graphs and nanostructures derived from thorn graphs. Let $P_{n}, S_{n}$, and $C_{n}$ denote the $n$-vertex path, star and cycle, respectively. It is easy to see that

$$
\operatorname{Gut}\left(P_{n}\right)=\frac{(n-1)\left(2 n^{2}-4 n+3\right)}{3}, \operatorname{Gut}\left(S_{n}\right)=(n-1)(2 n-3), \operatorname{Gut}\left(C_{n}\right)= \begin{cases}\frac{n\left(n^{2}-1\right)}{2} & \text { if } n \text { is odd }, \\ \frac{n^{3}}{2} & \text { if } n \text { is even. }\end{cases}
$$

\section{Thorn paths}

The thorn path $P_{n, p, k}$ is obtained from the path $P_{n}$ by adding $p$ neighbors to each of its nonterminal vertices and $k$ neighbors to each of its terminal vertices (see Fig. 3). Consider the path $P_{n}$ and choose a labeling for its vertices such that its two terminal vertices have numbers 1 and $n$ and its nonterminal vertices have numbers $2,3, \ldots, n-1$ as shown in Fig. 3. Then, $P_{n, p, k}$ can be considered as the thorn graph $\left(P_{n}\right)_{P}$, where $P$ is the $n$-tuple $\mathrm{P}=(k, p, \ldots, p, k)$. Using Eq. (1), we get the following theorem.

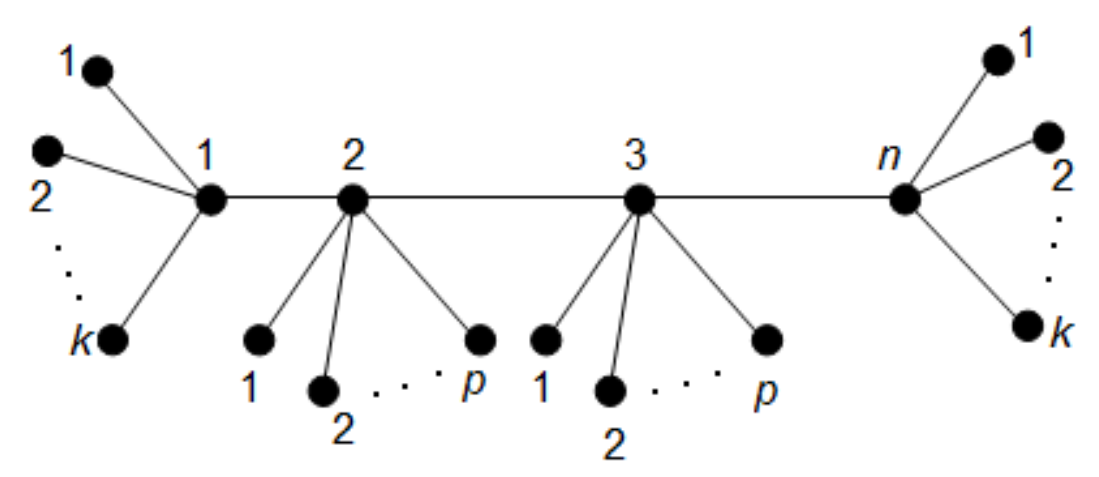

Figure 3. The thorn path $P_{n, p, k}$.

Theorem 3. Let $n \geq 2$ and let $p$ and $k$ be any nonnegative integers. Then

$$
\begin{aligned}
\operatorname{Gut}\left(P_{n, p, k}\right)= & \frac{(n-1)\left(2 n^{2}-4 n+3\right)}{3}+\frac{2 p^{2}}{3}\left(n^{3}-3 n^{2}-n+6\right)+\frac{p}{3}\left(4 n^{3}-12 n^{2}+5 n+6\right) \\
& +4 k^{2}(n+1)+2 k\left(2 n^{2}-2 n-1\right)+4 k p(n-2)(n+1) .
\end{aligned}
$$

\section{Thorn rods}

The thorn rod $P_{n, m}$ is a graph which includes a linear chain (termed "rod") of $n$ vertices and degree- $m$ terminal vertices at each of the two rod ends, where $m \geq 2$ (see Fig. 4). It is easy to see that $P_{n, m} \cong P_{n, 0, m-1}$. Using Eq. (7), we get the following corollary. 


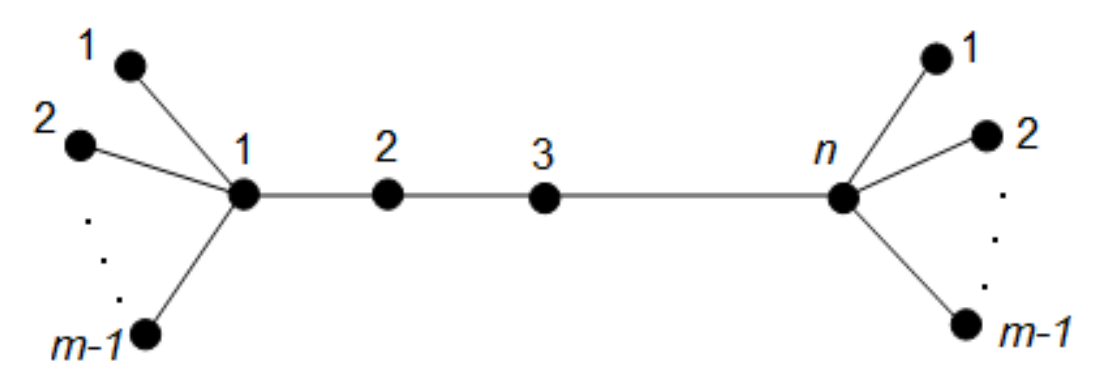

Figure 4. The thorn rod $P_{n, m}$.

Corollary 6. Let $n, m \geq 2$. Then

$$
\operatorname{Gut}\left(P_{n, m}\right)=\frac{(n-1)\left(2 n^{2}-4 n+3\right)}{3}+4(m-1)^{2}(n+1)+2(m-1)\left(2 n^{2}-2 n-1\right) .
$$

\section{Caterpillars}

The caterpillar $T^{*}(m, n)$ is a thorn graph whose parent graph is the path $P_{n}$ and whose $n$ nonterminal vertices are of the same degree $m>2$ (see Fig. 5). It is easy to see that $T^{*}(m, n) \cong P_{n, m-2, m-1}$. Using Eq. (7), we get the following corollary.

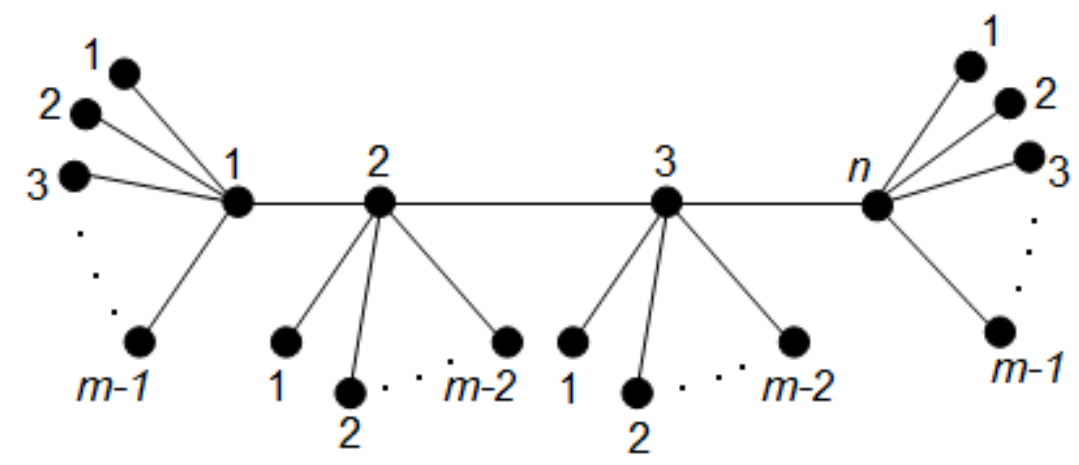

Figure 5. The caterpillar $T^{*}(m, n)$.

Corollary 7. Let $n \geq 2$ and $m>2$. Then

$$
\begin{aligned}
\operatorname{Gut}\left(T^{*}(m, n)\right)= & \frac{(n-1)\left(2 n^{2}-4 n+3\right)}{3}+\frac{2(m-2)^{2}}{3}\left(n^{3}-3 n^{2}-n+6\right)+\frac{m-2}{3}\left(4 n^{3}-12 n^{2}+5 n+6\right) \\
& +4(m-1)^{2}(n+1)+2(m-1)\left(2 n^{2}-2 n-1\right)+4(m-1)(m-2)(n-2)(n+1)
\end{aligned}
$$

\section{Thorn rings}

The $m$-thorn ring $C_{n, m}$ has a cycle $C_{n}$ as the parent, and $m-2$ thorns at each cycle vertex, where $m>2$. The 3 -thorn ring $C_{6,3}$ is depicted in Fig. 6. The $m$-thorn ring $C_{n, m}$ can be considered as the thorn graph $\left(C_{n}\right)_{P}$, where $P$ is the $n$-tuple $\mathrm{P}=(m-2, m-2, \ldots, m-2)$. Using Eq. (5) and the fact that $\operatorname{Gut}\left(C_{n}\right)=D D\left(C_{n}\right)=4 W\left(C_{n}\right)$, we get the following theorem. 


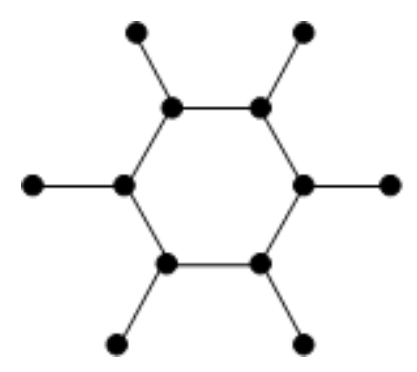

Figure 6. The 3-thorn ring $C_{6,3}$.

Theorem 4. Let $n, m \geq 3$. Then

$$
\operatorname{Gut}\left(C_{n, m}\right)= \begin{cases}\frac{n\left(n^{2}-1\right)}{2}(m-1)^{2}+n(m-2)(2 n(m-1)-1) & n \text { is odd }, \\ \frac{n^{3}}{3}(m-1)^{2}+n(m-2)(2 n(m-1)-1) & n \text { is even } .\end{cases}
$$

\section{Thorn stars}

The thorn star $S_{n, p, k}$ is obtained from the star $S_{n}$ by adding $p$ neighbors to the center of the star and $k$ neighbors to its terminal vertices (see Fig. 7). Consider the star $S_{n}$ and choose a labeling for its vertices such that its terminal vertices have numbers $1,2, \ldots, n-1$ and its central vertex has number $n$ as shown in Fig. 7. Then, $S_{n, p, k}$ can be considered as the thorn graph $\left(S_{n}\right)_{P}$, where $P$ is the $n$-tuple $\mathrm{P}=(k, k, \ldots, k, p)$. Using Eq. (1), we get the following theorem.

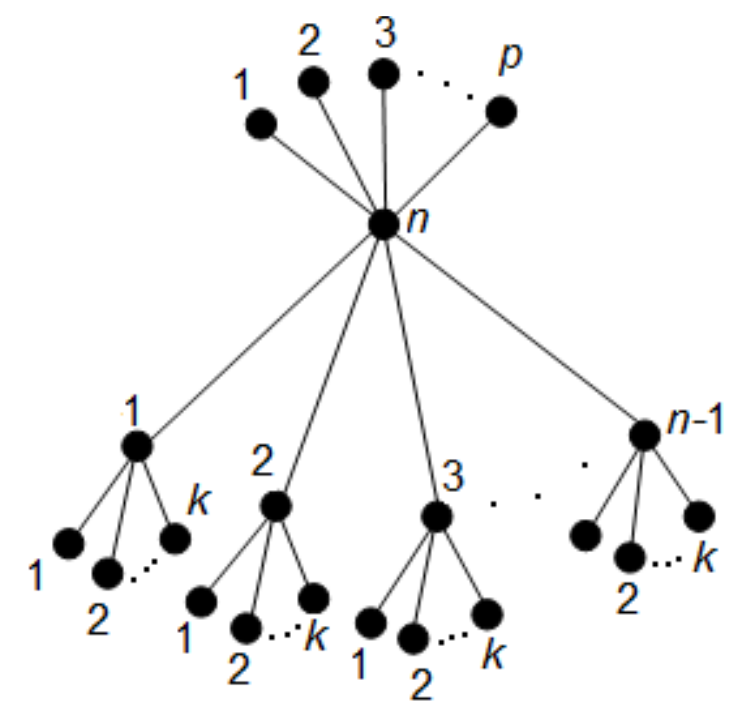

Figure 7. The thorn star $S_{n, p, k}$.

Theorem 5. Let $n \geq 3$ and let $p$ and $k$ be any nonnegative integers. Then

$$
\operatorname{Gut}\left(S_{n, p, k}\right)=(n-1)(2 n-3)+k\left(8 n^{2}-21 n+13\right)+2 k^{2}\left(3 n^{2}-8 n+5\right)
$$




$$
+8 p k(n-1)+p(4 n-5)+2 p^{2} .
$$

By setting $p=0$ in Eq. (8), we get the following corollary.

Corollary 8. Let $n \geq 3$ and let $k$ be any nonnegative integer. Then

$$
\operatorname{Gut}\left(S_{n, 0, k}\right)=(n-1)(2 n-3)+k\left(8 n^{2}-21 n+13\right)+2 k^{2}\left(3 n^{2}-8 n+5\right) .
$$

Consider the star graph $S_{n}$ and choose a labeling for its vertices such that its terminal vertices have numbers $1,2, \ldots, n-1$ and its central vertex has number $n$. Let $S_{n}\left(p_{1}, p_{2}, \ldots, p_{n-1}\right)$ denote the thorn star obtained by attaching $p_{i}$ terminal vertices to the vertex $i$ of $S_{n}$ for $i=1,2, \ldots, n-1$ (see Fig. 8). Using Eq. (1), we get the following theorem.

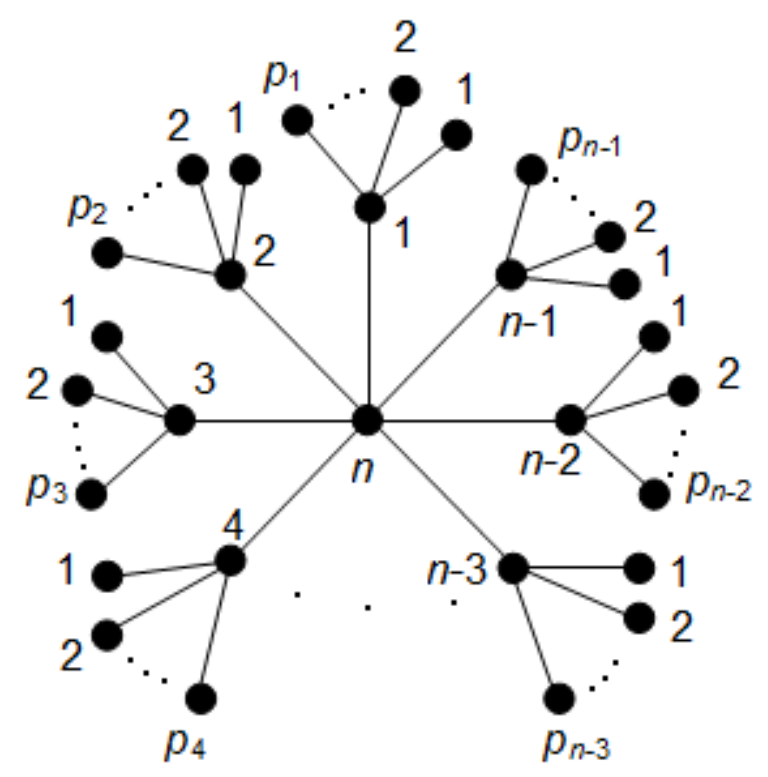

Figure 8. The thorn star $S_{n}\left(p_{1}, p_{2}, \ldots, p_{n-1}\right)$.

Theorem 6. Let $n \geq 3$ and let $p_{1}, p_{2} \ldots, p_{n-1}$ be nonnegative integers. Then

$$
\operatorname{Gut}\left(S_{n}\left(p_{1}, p_{2}, \ldots, p_{n-1}\right)\right)=(n-1)(2 n-3)+6\left(\sum_{i=1}^{n-1} p_{i}\right)^{2}-4 \sum_{i=1}^{n-1} p_{i}{ }^{2}+(8 n-13) \sum_{i=1}^{n-1} p_{i} \text {. (9) }
$$

\section{Kragujevac trees}

Let $P_{3}$ be the 3-vertex path rooted at one of its terminal vertices. For $k=2,3, \ldots$, construct the rooted tree $B_{k}$ by identifying the roots of $k$ copies of $P_{3}$. The vertex obtained by identifying the roots of $P_{3}$-trees is the root of $B_{k}$. Examples illustrating the structure of the rooted tree $B_{k}$ are depicted in Fig. 9. 


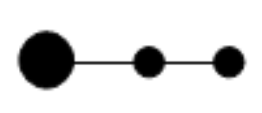

$P_{3}$

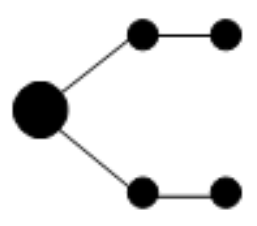

$B_{2}$

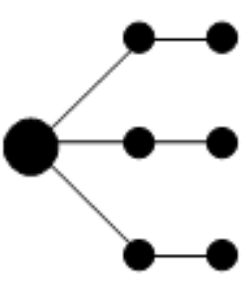

$B_{3}$

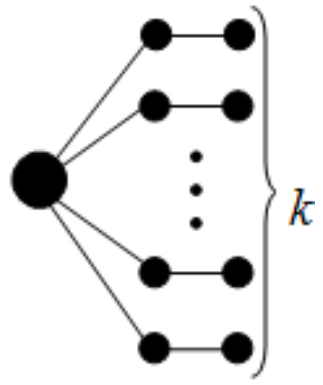

$B_{k}$

Figure 9 . The rooted trees $B_{2}, B_{3}$, and $B_{k}$. Their roots are indicated by large dots.

According to Gutman (2014), a Kragujevac tree $T$ is a tree possessing a vertex of degree $d \geq 2$, adjacent to the roots of $B_{p_{1}}, B_{p_{2}}, \ldots, B_{p_{d}}$, where $p_{1}, p_{2}, \ldots, p_{d} \geq 2$. This vertex is said to be the central vertex of $T$, whereas $d$ is the degree of $T$. The subgraphs $B_{p_{1}}, B_{p_{2}}, \ldots, B_{p_{d}}$ are the branches of $T$. Recall that some (or all) branches of $T$ may be mutually isomorphic. We denote the Kragujevac tree of degree $d$ with branches $B_{p_{1}}, B_{p_{2}}, \ldots, B_{p_{d}}$ by $K g\left(p_{1}, p_{2}, \ldots, p_{d}\right)$. A typical Kragujevac tree is depicted in Fig. 10.

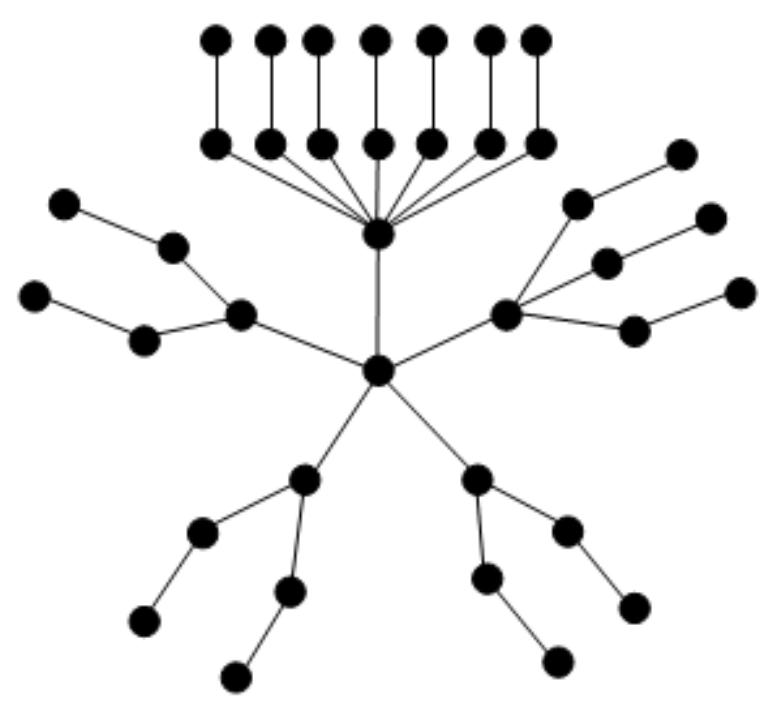

Figure 10. The Kragujevac tree $\operatorname{Kg}(7,3,2,2,2)$.

Theorem 7. The Gutman index of the Kragujevac tree $K g\left(p_{1}, p_{2}, \ldots, p_{d}\right)$ is given by

$$
\operatorname{Gut}\left(K g\left(p_{1}, p_{2}, \ldots, p_{d}\right)\right)=d(2 d-1)+32\left(\sum_{i=1}^{d} p_{i}\right)^{2}-16 \sum_{i=1}^{d}{p_{i}}^{2}+(20 d-18) \sum_{i=1}^{d} p_{i} .
$$

Proof. The Kragujevac tree $K g\left(p_{1}, p_{2}, \ldots, p_{d}\right)$ can be considered as the thorn graph obtained from the thorn star $S_{d+1}\left(p_{1}, p_{2}, \ldots, p_{d}\right)$ by attaching a pendent vertex to its pendent vertices. By setting $G=S_{d+1}\left(p_{1}, p_{2}, \ldots, p_{d}\right), p=1, k=\sum_{i=1}^{d} p_{i}$, and $|E(G)|=\sum_{i=1}^{d} p_{i}+d$ in Eq. (6), we obtain

$$
\operatorname{Gut}\left(K g\left(p_{1}, p_{2}, \ldots, p_{d}\right)\right)=\operatorname{Gut}\left(S_{d+1}\left(p_{1}, p_{2}, \ldots, p_{d}\right)\right)+8 T W\left(S_{d+1}\left(p_{1}, p_{2}, \ldots, p_{d}\right)\right)
$$




$$
+2 \Psi\left(S_{d+1}\left(p_{1}, p_{2}, \ldots, p_{d}\right)\right)+\left(\sum_{i=1}^{d} p_{i}\right)\left(4 \sum_{i=1}^{d} p_{i}+2 d-1\right) .
$$

By Eq. (9), we have

$$
\operatorname{Gut}\left(S_{d+1}\left(p_{1}, p_{2}, \ldots, p_{d}\right)\right)=d(2 d-1)+6\left(\sum_{i=1}^{d} p_{i}\right)^{2}-4 \sum_{i=1}^{d} p_{i}{ }^{2}+(8 d-5) \sum_{i=1}^{d} p_{i} .
$$

One can easily check that,

$$
\begin{aligned}
T W\left(S_{d+1}\left(p_{1}, p_{2}, \ldots, p_{d}\right)\right) & =\sum_{i=1}^{d} 2\left(\begin{array}{c}
p_{i} \\
2
\end{array}\right)+\sum_{1 \leq i<j \leq d} 4 p_{i} p_{j}=\sum_{i=1}^{d} p_{i}{ }^{2}-\sum_{i=1}^{d} p_{i}+2\left[\left(\sum_{i=1}^{d} p_{i}\right)^{2}-\sum_{i=1}^{d} p_{i}{ }^{2}\right] \\
& =2\left(\sum_{i=1}^{d} p_{i}\right)^{2}-\sum_{i=1}^{d} p_{i}{ }^{2}-\sum_{i=1}^{d} p_{i}, \\
\Psi\left(S_{d+1}\left(p_{1}, p_{2}, \ldots, p_{d}\right)\right) & =\sum_{i=1}^{d}\left(p_{i}+1\right)\left[p_{i} \times 1+3\left(\sum_{j=1}^{d} p_{j}-p_{i}\right)\right]+2 d \sum_{i=1}^{d} p_{i} \\
& =\sum_{i=1}^{d}\left(3 p_{i} \sum_{j=1}^{d} p_{j}-2 p_{i}{ }^{2}+3 \sum_{j=1}^{d} p_{j}-2 p_{i}\right)+2 d \sum_{i=1}^{d} p_{i} \\
& =3\left(\sum_{i=1}^{d} p_{i}\right)^{2}-2 \sum_{i=1}^{d} p_{i}{ }^{2}+(5 d-2) \sum_{i=1}^{d} p_{i} .
\end{aligned}
$$

Substituing the above formulae in Eq. (11), we can get Eq. (10).

\section{Dendrimers}

Let $D_{0}$ be the graph depicted in Fig. 11 .

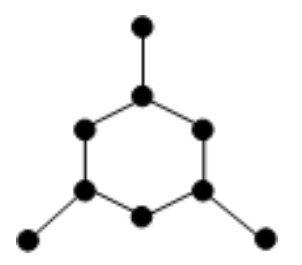

Figure 11. The dendrimer graph $\mathrm{D}_{0}$.

For positive integers $p$ and $h$, let $D_{p, h}$ be a series of dendrimers obtained by attaching $p$ pendent vertices to each pendent vertex of $D_{p, h-1}$ and let $D_{p, 0}=D_{0}$. We can also introduce the $D_{p, h}$ as the thorn graph obtained by attaching $p$ pendent vertices to each pendent vertex of $D_{p, h-1}$. This molecular structure can be encountered in real chemistry, e.g. in some tertiary phosphine dendrimers. Some examples of this kind of dendrimers are shown in Fig. 12. 


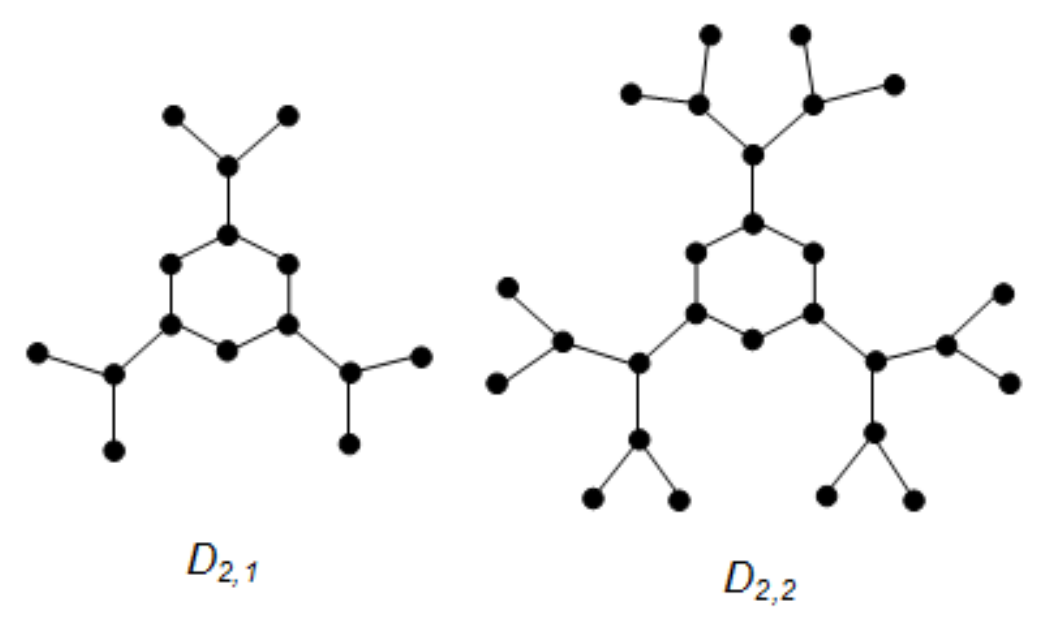

Figure 12. The dendrimer graphs $D_{p, h}$, for $p=2$ and $h=1,2$.

For a fixed positive integer $p$, let $k_{h}$ denote the number of pendent vertices of $D_{p, h}$, $h \geq 0$. Obviously, $k_{h}=p k_{h-1}$ and $\left|E\left(D_{p, h}\right)\right|=\left|E\left(D_{p, h-1}\right)\right|+3 p^{h}$. So for every $h \geq 0$, we have

$$
k_{h}=3 p^{h},\left|E\left(D_{p, h}\right)\right|=6+3 \sum_{i=0}^{h} p^{i} .
$$

It is easy to check that $T W\left(D_{0}\right)=12$. In (AZARI and IRANMANESH, 2016), an explicit formula for computing the terminal Wiener index of the dendrimer graph $D_{p, h}$ was computed.

Theorem 8. (Azari and Iranmanesh, 2016) Let $p$ and $h$ be positive integers. The terminal Wiener index of the dendrimer graph $D_{p, h}$ is given by

$$
T W\left(D_{p, h}\right)=(9 h+12) p^{2 h}-3 p^{h} \sum_{i=0}^{h-1} p^{i} \text {. }
$$

It is easy to check that $\Psi\left(D_{0}\right)=111$ and $\operatorname{Gut}\left(D_{0}\right)=291$. In the following theorem, we present recurrence relations for computing $\Psi\left(D_{p, h}\right)$ and $\operatorname{Gut}\left(D_{p, h}\right)$. Results are deduced from Eqs. (4) and (6), and the proof of the theorem is therefore omitted.

Theorem 9. Let $p$ and $h$ be positive integers. Then

$$
\begin{aligned}
& \Psi\left(D_{p, h}\right)=2 p(p+1) T W\left(D_{p, h-1}\right)+p \Psi\left(D_{p, h-1}\right)+3 p^{h}\left(3 p^{h}+12+6 \sum_{i=0}^{h-1} p^{i}\right), \\
& \operatorname{Gut}\left(D_{p, h}\right)=\operatorname{Gut}\left(D_{p, h-1}\right)+4 p(p+1) T W\left(D_{p, h-1}\right)+2 p \Psi\left(D_{p, h-1}\right)+3 p^{h}\left(11+6 \sum_{i=0}^{h} p^{i}\right) .
\end{aligned}
$$

Using Eqs. (12)-(14), we can compute the Gutman index of the dendrimer graph $D_{p, h}$ for every positive integers $p$ and $h$.

For example, by setting $h=1$ in Eqs. (12)-(14), we get $T W\left(D_{p, 1}\right)=21 p^{2}-3 p$, $\Psi\left(D_{p, 1}\right)=2 p(p+1) T W\left(D_{0}\right)+p \Psi\left(D_{0}\right)+3 p(3 p+12+6)=33 p^{2}+189 p$, 
$\operatorname{Gut}\left(D_{p, 1}\right)=\operatorname{Gut}\left(D_{0}\right)+4 p(p+1) T W\left(D_{0}\right)+2 p \Psi\left(D_{0}\right)+3 p[11+6(1+p)]=66 p^{2}+321 p+291$.

By setting $h=2$ in Eqs. (12)-(14), we get

$$
\begin{aligned}
T W\left(D_{p, 2}\right) & =30 p^{4}-3 p^{3}-3 p^{2} \\
\Psi\left(D_{p, 2}\right)= & 2 p(p+1) T W\left(D_{p, 1}\right)+p \Psi\left(D_{p, 1}\right)+3 p^{2}\left[3 p^{2}+12+6(1+p)\right]=51 p^{4}+87 p^{3}+237 p^{2} \\
\operatorname{Gut}\left(D_{p, 2}\right) & =\operatorname{Gut}\left(D_{p, 1}\right)+4 p(p+1) T W\left(D_{p, 1}\right)+2 p \Psi\left(D_{p, 1}\right)+3 p^{2}\left[11+6\left(1+p+p^{2}\right)\right] \\
& =102 p^{4}+156 p^{3}+483 p^{2}+321 p+291 .
\end{aligned}
$$

By setting $h=3$ in Eqs. (12)-(14), we get

$$
\begin{aligned}
& T W\left(D_{p, 3}\right)=39 p^{6}-3 p^{5}-3 p^{4}-3 p^{3} \\
& \begin{aligned}
\Psi\left(D_{p, 3}\right)= & 2 p(p+1) T W\left(D_{p, 2}\right)+p \Psi\left(D_{p, 2}\right)+3 p^{3}\left[3 p^{3}+12+6\left(1+p+p^{2}\right)\right] \\
= & 69 p^{6}+123 p^{5}+93 p^{4}+285 p^{3} \\
\operatorname{Gut}\left(D_{p, 3}\right) & =\operatorname{Gut}\left(D_{p, 2}\right)+4 p(p+1) T W\left(D_{p, 2}\right)+2 p \Psi\left(D_{p, 2}\right)+3 p^{3}\left[11+6\left(1+p+p^{2}+p^{3}\right)\right] \\
& =138 p^{6}+228 p^{5}+270 p^{4}+669 p^{3}+483 p^{2}+321 p+291 .
\end{aligned}
\end{aligned}
$$

By setting $h=4$ in Eqs. (12)-(14), we get

$$
\begin{aligned}
& T W\left(D_{p, 4}\right)=48 p^{8}-3 p^{7}-3 p^{6}-3 p^{5}-3 p^{4}, \\
& \Psi\left(D_{p, 4}\right)=2 p(p+1) T W\left(D_{p, 3}\right)+p \Psi\left(D_{p, 3}\right)+3 p^{4}\left[3 p^{4}+12+6\left(1+p+p^{2}+p^{3}\right)\right] \\
& =87 p^{8}+159 p^{7}+129 p^{6}+99 p^{5}+333 p^{4}, \\
& \operatorname{Gut}\left(D_{p, 4}\right)=\operatorname{Gut}\left(D_{p, 3}\right)+4 p(p+1) T W\left(D_{p, 3}\right)+2 p \Psi\left(D_{p, 3}\right)+3 p^{4}\left[11+6\left(1+p+p^{2}+p^{3}+p^{4}\right)\right] \\
& =174 p^{8}+300 p^{7}+378 p^{6}+408 p^{5}+879 p^{4}+669 p^{3}+483 p^{2}+321 p+291 .
\end{aligned}
$$

The Gutman index of $D_{p, h}$ for $p=2,3$ and $h \leq 4$ is computed in Tab. 1 .

Table 1. The Gutman index of the dendrimer graphs $D_{p, h}$ for $p=2,3$ and $h \leq 4$.

\begin{tabular}{ccc}
\hline h & Gut(D, & Gut $\left(\mathbf{D}_{\mathbf{3}, \mathbf{h}}\right)$ \\
\hline $\mathbf{0}$ & 291 & 291 \\
$\mathbf{1}$ & 1197 & 1848 \\
$\mathbf{2}$ & 5745 & 18075 \\
$\mathbf{3}$ & 28665 & 201540 \\
$\mathbf{4}$ & 142473 & 2267283 \\
\hline
\end{tabular}

\section{Acknowledgments}

The author would like to thank the referee for insightful comments. 


\section{References:}

[1] Alizadeh, Y., Iranmanesh, A., Došlić, T., Azari, M. (2014): The edge Wiener index of suspensions, bottlenecks, and thorny graphs. Glas. Mat. Ser. III 49 (69): 1-12. doi: 10.3336/gm.49.1.01

[2] Andova, V., Dimitrov, D., Fink, J., ŠKrekovski, R. (2012): Bounds on Gutman index. MATCH Commun. Math. Comput. Chem. 67: 515-524.

[3] AzARI, M. (2014): Sharp lower bounds on the Narumi-Katayama index of graph operations. Appl. Math. Comput. 239: 409-421. doi: 10.1016/j.amc.2014.04.088

[4] AzARI, M. (2016): Some results on vertex version and edge versions of modified Schultz index. Int. J. Math. Combin. 2: 65-82.

[5] Azari, M., Iranmanesh, A. (2015): Clusters and various versions of Wiener-type invariants. Kragujevac J. Math. 39 (2): 155-171. doi: 10.5937/KgJMath1502155A

[6] Azari, M., Iranmanesh, A. (2016): Dendrimer graphs as thorn graphs and their topological edge properties. Natl. Acad. Sci. Lett. 39 (6): 455-460. doi: 10.1007/s40009016-0514-5

[7] Azari, M., Divanpour, H. (2017): Splices, links, and their edge-degree distances. Trans. Comb. 6 (4): 29-42. doi: 10.22108/toc.2017.21614

[8] Bonchev, D., Klein, D.J. (2002): On the Wiener number of thorn trees, stars, rings and rods. Croat. Chem. Acta, 75 (2): 613-620.

[9] Bytautas, L., Bonchev, D., Klein, D.J. (2001): On the generation of mean Wiener numbers of thorny graphs. MATCH Commun. Math. Comput. Chem. 44: 31-40.

[10] Cayley, A. (1874): On the mathematical theory of isomers. Phil. Magazine, 47: 444447. doi: 10.1080/14786447408641058

[11] Chen, S. (2016): Cacti with the smallest, second smallest, and third smallest Gutman index. J. Comb. Optim. 31 (1): 327-332. doi: 10.1007/s10878-014-9743-z

[12] DiUdeA, M.V. (2001): QSPR/QSAR Studies by Molecular Descriptors. Nova, New York.

[13] Dobrynin, A., Kochetova, A.A. (1994): Degree distance of a graph: A degree analogue of the Wiener index. J. Chem. Inf. Comput. Sci. 34 (5): 1082-1086.

[14] Feng, L., LIU, W. (2011): The maximal Gutman index of bicyclic graphs. MATCH Commun. Math. Comput. Chem. 66: 669-708.

[15] Gutman, I. (1994): Selected properties of the Schultz molecular topological index. $J$. Chem. Inf. Comput. Sci. 34 (5): 1087-1089.

[16] Gutman, I. (1998): Distance in thorny graph. Publ. Inst. Math. (Beograd) 63: 31-36.

[17] Gutman, I. (2014): Kragujevac trees and their energy. SER A: Appl. Math. Inform. and Mech. 6 (2): 71-79.

[18] Gutman, I. (2016): On two degree-and-distance-based graph invariants. Bull. Acad. Serbe Sci. Arts (Cl. Sci. Math. Natur.) 149 (41): 21-31. 
[19] Gutman, I., Vidović, D., Popović, LJ. (1998): Graph representation of organic molecules-Cayley's plerograms vs. his kenograms. J. Chem. Soc. Faraday Trans. 94: 857-860. doi: 10.1039/A708076J

[20] Gutman, I., Furtula, B., Petrović, M. (2009): Terminal Wiener index. J. Math. Chem. 46: 522-531. doi: 10.1007/s10910-008-9476-2

[21] Heydari, A., Gutman, I. (2010): On the terminal Wiener index of thorn graphs. Kragujevac J. Sci. 32: 57-64.

[22] Klein, D.J., DošLić, T., BoncheV, D. (2007): Vertex-weightings for distance moments and thorny graphs, Discrete Appl. Math. 155 (17): 2294-2302. doi: 10.1016/j.dam.2007.05.042

[23] Knor, M., PotoČNiK, P., ŠKrekovski, R. (2014): Relationship between the edgeWiener index and the Gutman index of a graph. Discrete Appl. Math. 167: 197-201. doi: 10.1016/j.dam.2013.12.009

[24] LI, S. (2011): Zagreb polynomials of thorn graphs. Kragujevac J. Sci. 33: 33-38.

[25] Polya, G. (1937): Kombinatorische Anzahlbestimmungen fur Gruppen, Graphen und chemische Verbindungen. Acta Math. 68: 145-254. doi: 10.1007/BF02546665

[26] SzÉKELy, L.A., WANG, H., WU, T. (2011): The sum of distances between the leaves of a tree and the 'semi-regular' property. Discrete Math. 311 (13): 1197-1203. doi: 10.1016/j.disc.2010.06.005

[27] VukiČEvić, D., Graovac, A. (2004): On modified Wiener indices of thorn graphs. MATCH Commun. Math. Comput. Chem. 50: 93-108.

[28] Vukičević, D., Nikolić, S., Trinajstić, N. (2005): On the Schultz index of thorn graphs. Internet Electron. J. Mol. Des. 4: 501-514.

[29] VukičEvić, D., Zhou, B., Trinajstić, N. (2007): Altered Wiener indices of thorn trees. Croat. Chem. Acta, 80: 283-285.

[30] Walikar, H.B., Ramane, H.S., Sindagi, L., Shirakol, S.S., Gutman, I. (2006): Hosoya polynomial of thorn trees, rods, rings, and stars. Kragujevac J. Sci. 28: 47-56.

[31] WiEner, H. (1947): Structural determination of paraffin boiling points. J. Am. Chem. Soc. 69 (1): 17-20. doi: 10.1021/ja01193a005

[32] Zhou, B. (2005): On modified Wiener indices of thorn trees. Kragujevac J. Math. 27: 5-9. 